\title{
Resistência ao cisalhamento de ligações de madeira para produção de painel do sistema wood frame com eucalipto jovem
}

\author{
Shear strength of timber joints for stud members \\ for framing construction produced with juvenile \\ eucalyptus wood
}

\author{
Filipe Luigi Dantas Lima Santos ${ }^{1}$, Júlia Cruz da Silva ${ }^{1}$, \\ Rita Dione Araújo Cunha ${ }^{2}$, Alexandre de Macêdo Wahrhaftig ${ }^{3}$, \\ Sandro Fábio César ${ }^{3}$
}

\footnotetext{
${ }^{1}$ Programa de Pós-graduação em Engenharia Civil (PPEC), Universidade Federal da Bahia (UFBA), Escola Politécnica, Rua Prof. Aristídes Novis, 2, Federação, Salvador, Bahia, Brasil.

${ }^{2}$ Núcleo de Tecnologia, Projeto e Planejamento, Universidade Federal da Bahia (UFBA), Faculdade de Arquitetura e Urbanismo, Rua Caetano Moura, 121, Federação, Salvador, Bahia, Brasil.

${ }^{3}$ Departamento de Construção e Estrutura, Universidade Federal da Bahia (UFBA), Escola Politécnica, Rua Prof. Aristídes Novis, n. 2, Federação, Salvador, Bahia, Brasil.

e-mail: filipeluigi.s@gmail.com,jsjuliaria@gmail.com,ritadi@uol.com.br, alixa@ufba.br, sfcesarpaz@uol.com.br
}

\section{RESUMO}

Em estruturas de wood frame, as ligações entre montantes e banzos tem como finalidade garantir a rigidez e o contraventamento necessários para essas construções, que são naturalmente flexíveis, quando submetidas aos esforços horizontais tais como vento e sismos. Este trabalho teve como objetivo avaliar a resistência ao cisalhamento de ligações de madeira para produção de painel de wood frame produzidos com madeira de eucalipto jovem (menos que 15 anos de idade), tendo em vista que o plantio de espécies desse gênero é mais abundante no país. Os tipos de ligações escolhidas para este estudo foram do tipo chapa de dente estampado, parafuso autoatarraxante e encaixe de madeira. Para cumprir esse objetivo, foram utilizados corpos de prova de madeira de Eucalyptus pellita com idade variando entre 7 e 10 anos (classe C20). Foram utilizados os procedimentos experimentais previstos no projeto de revisão de norma da ABNT PN 02:126.10-001-4 (ABNT, 2017). Os resultados indicaram que a ligação do tipo encaixe é a mais apropriada para a ligação entre banzos e montantes de painéis de wood frame produzidos com madeira de eucalipto jovem, por ter melhor resistência e maior rigidez ao cisalhamento quando comparada com as ligações com parafuso autoatarraxante à $90^{\circ} \mathrm{e}$ chapa de dente estampado.

Palavras-chave: Ligações em madeira. Eucalipto jovem. Chapa de dente estampado. Parafuso autoatarraxante. Encaixe de madeira.

\footnotetext{
ABSTRACT

In wood frame structures, the frame connection plays an important role to guarantee stability for those constructions that are naturally flexible when subjected to shear during wind or earthquake. This work aimed to evaluate the shear strength of timber joints for wood frame shear wall produced with young eucalyptus wood (less than 15 years old), considering that the planting of species of this genus are more abundant in the country. The types of connections chosen for this study were metal plate, self-tapping screw, and rectangular mortise and tenon. To achieve this objective, 7 to 10 years old Eucalyptus pellita wood (class C20) was used to produce the specimens. The experimental procedures were provided by in the standard revision project ABNT PN 02:126.10-001-4 (ABNT, 2017). The results indicated that the mortise and tenon are the most suitable for frame to frame connection of wood frame shear wall due to its higher shear resistance and stiffness when compared to the self-tapping screw connected in $90^{\circ}$ angle and metal plate.
} 
Keywords: Timber joints. Young eucalyptus wood. Truss Connector Plate. Self-tapping screws. Mortise and tenon.

\section{INTRODUÇÃO}

A madeira é um dos materiais de construção mais utilizados no mundo, tendo dominado os métodos construtivos por séculos e deve ser utilizada de maneira compatível com suas propriedades. Desde seus primeiros usos, as técnicas de construção em madeira têm passado por transformações que visam principalmente a otimização dos recursos e a eficiência construtiva. Mais recentemente, dada as preocupações ambientais e os esforços empregados para a diminuição das emissões de carbono, os sistemas construtivos em madeira destacam-se por serem sustentáveis e pela capacidade de retenção de carbono, como informado por ARAÚJO [1].

Entretanto, edificações em madeira constituem sistemas não monolíticos e a rigidez dessas construções está diretamente associada aos materiais utilizados para a ligação que conectam os elementos [2]. O desempenho dessas ligações é influenciado pela espécie, pela presença ou não de defeitos na madeira, pela direção em que o conector está sendo aplicado em relação às fibras, por possíveis variações dimensionais que possam a ocorrer devido à mudança no teor de umidade e à temperatura do ambiente ou se a construção se encontra em situação de incêndio [3-6].

O estudo de ligações em madeira é um tema complexo e durante anos foram utilizados métodos empíricos para o dimensionamento desses elementos. A partir da segunda metade do século XX, surgiram teorias que passaram ser incorporadas ao projeto. Notoriamente, uma das técnicas mais conhecidas está associada à teoria que JOHANSEN [7] desenvolveu para a aplicação de conectores metálicos em elementos de madeira. Atualmente, LIU e XIONG [8] afirmam que os métodos definidos por normas para a caracterização de resistência e rigidez de ligações oferecem resultados satisfatórios, no entanto, salientam a necessidade de maior investigação para aproximar a teoria do comportamento real de cada tipo de estrutura.

A norma brasileira de dimensionamento em madeira, ABNT NBR 7190 - Projeto de Estruturas de Madeira [9], preconiza que as ligações mecânicas podem ser feitas de três formas: por pinos metálicos, sendo divididas entre pregos e parafusos; por pinos de madeiras ou por conectores metálicos, sendo utilizados anéis ou chapas metálicas. Vale lembrar que a norma brasileira foi concebida num momento em que era mais comum o uso de madeira adulta de cerne de espécies de alta densidade para construções, especialmente de coberturas $[10,11]$. Sendo assim, constata-se uma lacuna para aplicação de madeira jovem de espécies oriundas de florestas plantadas.

Durante os anos, com o desenvolvimento da indústria florestal, passou-se a produzir madeira serrada proveniente de árvores de pequeno diâmetro com baixo ciclo de espécies exóticas. Por essa razão, busca-se, nesta investigação, avaliar a adequação de conceitos normativos previamente desenvolvidos a esta condição de uso. Poucos trabalhos foram conduzidos para determinar as propriedades de materiais para ligação de madeira oriundas de árvores jovens, com fim de verificar a utilização dessas espécies em sistemas construtivos industrializados modernos no Brasil [6, 12, 13], como o wood frame.

A estabilidade de estruturas de wood frame para cargas horizontais depende da capacidade lateral da parede diafragma [14] feita pela ossatura de madeira fechada com chapa de Oriented Stand Board (OSB), sendo que esta capacidade lateral da parede depende da resistência ao cisalhamento da chapa OSB e das ligações que conectam os banzos e montantes que compõem a ossatura de madeira [15]. Outros autores buscaram avaliar o comportamento de ligações entre banzos e montantes, quando buscaram adaptar os painéis de sistema wood frame para materiais disponíveis no mercado local [16, 17]. SILVA [18] publicou um projeto conceitual de produção de painéis de wood frame utilizando nos montantes e banzos o eucalipto jovem (Eucalyptus spp.), tendo em vista que as espécies desse gênero são mais abundantes no país [19]. No referido trabalho, a autora propõe que árvores jovens (com idade entre 7 e 10 anos) com diâmetro médio de $12 \mathrm{~cm}$ no topo da tora podem ser cortadas para uma seção de $9,0 \mathrm{~cm}$ x $9,0 \mathrm{~cm}$ e utilizadas para produção dos painéis. A seção proposta pela autora tem presença de cerne, alburno, madeira juvenil e medula.

No presente trabalho estudaram-se as ligações entre montantes e banzos para esta configuração proposta por SILVA [18]. Dessa forma, o objetivo desta pesquisa foi avaliar a resistência ao cisalhamento de ligações de madeira entre elementos horizontais e verticais que compõe a ossatura do painel de wood frame produzido com madeira de eucalipto jovem. 


\section{MATERIAIS E MÉTODOS}

\section{Identificação da madeira}

Esta pesquisa foi realizada com madeira de Eucalyptus pellita, com idades entre 7 e 10 anos, originadas da Fazenda São Marcos, situado no município de Araçás, no Estado da Bahia, Brasil. As propriedades físicas e mecânicas da madeira foram determinadas segundo os métodos presentes no Anexo B da norma brasileira ABNT NBR 7190 [9] e são apresentadas na Tabela 1, onde $\mathrm{N}$ representa o número de corpos de prova, $\mathrm{f}_{\mathrm{c} 0}$, $\mathrm{f}_{\mathrm{c} 0, \mathrm{k}} \mathrm{f}_{\mathrm{v} 0, \mathrm{k}}$ são a resistência média e característica à compressão paralela às fibras e a resistência ao cisalhamento; $\mathrm{E}_{\mathrm{c} 0,12 \%}$ é o módulo de elasticidade na compressão paralela às fibras; $\rho_{\mathrm{bas}}$ e $\rho_{\mathrm{ap}}$ são a densidade básica e aparente, com todos os parâmetros relativos à umidade de $12 \%$.

Tabela 1: Propriedades físicas e mecânicas da madeira.

\begin{tabular}{l|c|c|c|c|c|c}
\hline \multicolumn{1}{c|}{ ESPÉCIE } & $\begin{array}{c}\mathbf{f}_{\mathrm{c} 0} \\
(\mathbf{M P a})\end{array}$ & $\begin{array}{c}\mathbf{f}_{\mathrm{c} 0, \mathbf{k}} \\
(\mathbf{M P a})\end{array}$ & $\begin{array}{c}\mathbf{f}_{\mathrm{v} 0, \mathbf{k}} \\
(\mathbf{M P a})\end{array}$ & $\begin{array}{c}\mathbf{E}_{\mathrm{c} 0,12 \%} \\
(\mathbf{M P a})\end{array}$ & $\begin{array}{c}\boldsymbol{\rho}_{\text {bas }} \\
\left(\mathbf{k g} / \mathbf{m}^{3}\right)\end{array}$ & $\begin{array}{c}\boldsymbol{\rho}_{\mathrm{ap}} \\
\left(\mathbf{k g} / \mathbf{m}^{3}\right)\end{array}$ \\
\hline $\mathrm{N}$ & 12 & 12 & 13 & 12 & 33 & 33 \\
\hline E. pellita & 50,66 & 41,98 & 8,07 & 12721,00 & 682,00 & 802,00 \\
\hline
\end{tabular}

$\mathrm{f}_{\mathrm{c} 0}$ : resistência média à compressão paralela às fibras a $12 \%$ de umidade; $\mathrm{f}_{\mathrm{c} 0, \mathrm{k}}$ : resistência característica à compressão paralela às fibras; $\mathrm{f}_{\mathrm{v} 0, \mathrm{k}}$ : resistência ao cisalhamento paralelo às fibras; $\mathrm{E}_{\mathrm{c} 0,12 \%}$ : módulo de elasticidade na compressão paralela às fibras; $\rho_{\text {bas }}$ : densidade básica; $\rho_{a p}$ : densidade aparente à $12 \%$ de umidade; N: quantidade de corpos de prova.

Os dados apresentados na Tabela 1 indicam que a madeira de E. pellita pode ser classificada como madeira de classe C40, tendo em vista que, segundo o item 10.6 da ABNT NBR 7190 [9], a caracterização da madeira deve ser feita segundo a resistência característica à compressão paralela às fibras da madeira $\left(\mathrm{f}_{\mathrm{c} 0, \mathrm{k}}\right)$. No entanto, por ser madeira jovem, a espécie não obteve módulo de elasticidade a compressão $\left(\mathrm{E}_{\mathrm{c} 0,12 \%}\right)$ como esperado para a classe C40 do grupo de dicotiledôneas, que se situa em torno de 19500,00 MPa. Sendo assim, optou-se por classificar a madeira estudada como classe C20 por ser a favor da segurança e cumprir os todos os requisitos de valores mínimos para as propriedades físico-mecânicas para essa classe.

Para a produção de montantes e banzos de painéis de wood frame é recomendado o uso de madeira com classificação mínima de classe C20 [20]. Desta forma, a madeira de E. pellita jovem teve comportamento físico-mecânico dentro do esperado para a produção de componentes estruturais para o sistema construtivo considerado no presente estudo.

\subsection{Descrição dos corpos de prova}

Para a confecção dos corpos de prova, as toras de madeira foram cortadas para gerar peças prismáticas de dimensões $90 \mathrm{~mm}$ x $90 \mathrm{~mm}$ x $300 \mathrm{~mm}$ em concordância com o trabalho de SILVA [18] e estocadas até umidade de equilíbrio, em torno de $17 \%$, esperada para regiões com umidade relativa do ar entre $75 \%$ e $85 \%$, como no caso da cidade de Salvador [9], onde a pesquisa foi realizada. A seção das peças tinha presença de medula, cerne, alburno e madeira juvenil.

Foi identificada pouca presença de rachaduras após a secagem, devido a boa estabilidade dimensional da madeira de Eucalyptus pellita, o que foi demonstrado previamente por SANTOS et al. [21]. Após a secagem, três peças foram unidas de acordo com a ligação selecionada, formando corpos de prova em forma de "H" de modo que a direção das fibras da madeira fosse perpendicular entre as peças. Foram estudados três tipos de ligações, sendo elas: chapas de dente estampado, parafusos autoatarraxantes e encaixes de madeira.

O ensaio com chapa de dente estampado foi feito com o uso de quatro chapas localizados nas faces de conexão, sendo assim, duas de cada lado do corpo de prova. Para este ensaio foram consideradas três configurações distintas das peças que faziam parte do corpo de prova:
a) grupo CDE-ACA - Peças ordenadas em: alburno - cerne - alburno (Figura 1a);
b) grupo CDE-CCC - Peças odenadas em: cerne - cerne - cerne (Figura 1b);
c) grupo CDE-AAA - Peças ordenadas em: alburno - alburno - alburno (Figura 1c).

A Figura 1 mostra os corpos de prova para o ensaio das ligações com chapa de dente estampado, destacando a cor mais escura para representar áreas de cerne e a cor mais clara para representar o alburno. Estas três combinações foram propostas, visando verificar a influência da densidade na região do alburno e do cer- 
ne na resistência de ligações com chapas de dente estampado, tendo em vista que este parâmetro é relevante para a resistência desse tipo de ligação.

A partir do ensaio de densidade, foi identificado que, para a madeira de Eucalyptus pellita jovem estudada, o cerne tem densidade aparente $21 \%$ maior que o alburno, sendo a densidade aparente do alburno de $716 \mathrm{~kg} / \mathrm{m}^{3}$ e a do cerne de $866 \mathrm{~kg} / \mathrm{m}^{3}$. As especificações da chapa de dente estampado estão presentes no Quadro 1. Para o ensaio com parafuso autoatarraxante foram produzidos corpos de prova com cravação a $90^{\circ}$ em relação ao montante (Figura 2a), sendo esse tipo de cravação escolhida com a intenção de reproduzir uma prática de obra de estruturas em wood frame, onde são preferidas as ligações de parafusos a $90^{\circ}$ às ligações com ângulo de $45^{\circ}$. Cada corpo de prova possuía dois parafusos. Foi feita uma pré-furação com $6 \mathrm{~mm}$ de diâmetro e $16 \mathrm{~cm}$ de comprimento, seguindo determinação do item 8.3.3 da ABNT NBR 7190 [9], onde se recomenda que, para árvores de dicotiledôneas, a pré-furação de parafusos deve ser $\mathrm{d}_{0}=0,98 \cdot \mathrm{d}_{\mathrm{ef}}$, onde $\mathrm{d}_{0}$ é o diâmetro do furo e $\mathrm{d}_{\mathrm{ef}}$ é o diâmetro do parafuso. Em seguida, os parafusos foram colocados com parafusadeira mecânica. A especificação dos parafusos está presente no Quadro 1.

Para o ensaio com encaixe de madeira foram considerados dois grupos com diferentes dimensões de comprimento do encaixe (Figura 2b):

a) grupo Encaixe C30 - Encaixe retangular de madeira tipo macho-fêmea com dimensões 30 $\mathrm{mm} \times 30 \mathrm{~mm} \times 30 \mathrm{~mm}$;

b) grupo Encaixe C45 - Encaixe retangular de madeira tipo macho-fêmea com dimensões 30 $\mathrm{mm} \times 30 \mathrm{~mm}$ x $45 \mathrm{~mm}$.

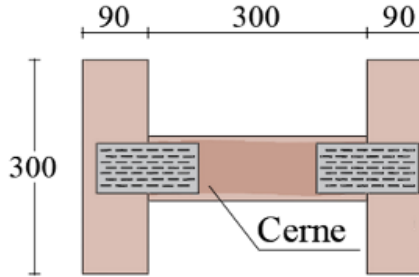

CDE-ACA

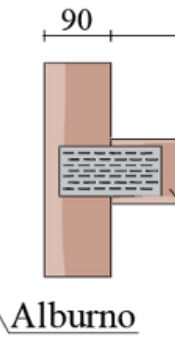

CDE-CCC

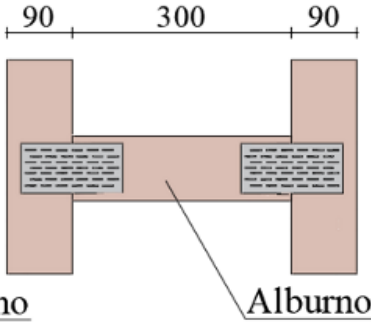

CDE-AAA

(a)

(b)

(c)

Figura 1: Representação dos corpos de prova das ligações com chapas de dente estampado.

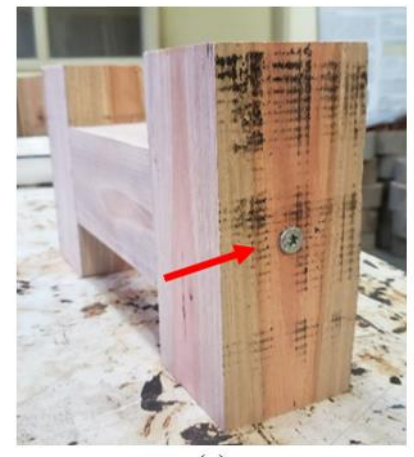

(a)

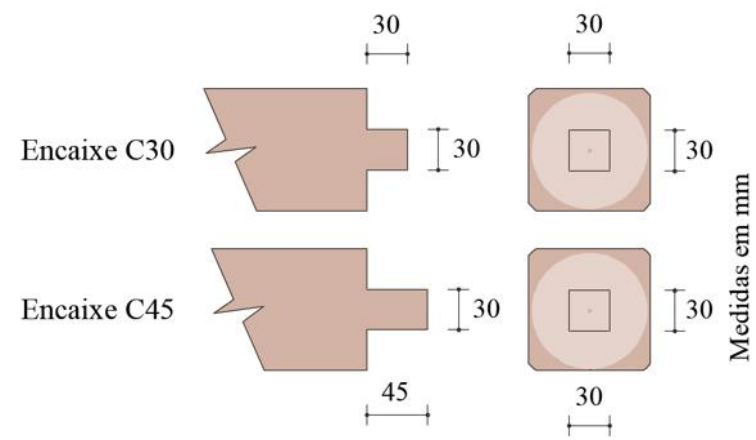

(b)

Figura 2: Arranjo de corpo de prova para ensaio de resistência ao cisalhamento das ligações: (a) corpo de prova com parafuso autoatarraxante; (b) dimensões de corpo de prova de encaixe. 
Quadro 1: Resumo dos ensaios e especificação das ligações.

\begin{tabular}{|c|c|c|c|c|}
\hline CÓDIGO & LIGAÇÃO & TIPO & $\mathbf{N}$ & ESPECIFICAÇÕES \\
\hline CDE-AAA & $\begin{array}{c}\text { Chapa de dente } \\
\text { estampado }\end{array}$ & $\begin{array}{l}\text { GANG NAIL® } \\
\text { GNA80 } \\
\end{array}$ & 5 & $\begin{array}{c}\text { D: } 71 \mathrm{~mm} \times 139 \mathrm{~mm} \text { x } 7,8 \mathrm{~mm} \\
\text { Densidade: } 1,4 \text { dentes } / \mathrm{cm}^{2}\end{array}$ \\
\hline CDE-ACA & $\begin{array}{c}\text { Chapa de dente } \\
\text { estampado }\end{array}$ & $\begin{array}{l}\text { GANG NAIL® } \\
\text { GNA80 } \\
\end{array}$ & 5 & $\begin{array}{c}\text { D: } 71 \mathrm{~mm} \text { x } 139 \mathrm{~mm} \times 7,8 \mathrm{~mm} \\
\text { Densidade: } 1,4 \text { dentes } / \mathrm{cm}^{2}\end{array}$ \\
\hline CDE-CCC & $\begin{array}{l}\text { Chapa de dente } \\
\text { estampado }\end{array}$ & $\begin{array}{l}\text { GANG NAIL® } \\
\text { GNA80 }\end{array}$ & 5 & $\begin{array}{l}\text { D: } 71 \mathrm{~mm} \text { x } 139 \mathrm{~mm} \text { x 7,8 mm } \\
\text { Densidade: } 1,4 \text { dentes } / \mathrm{cm}\end{array}$ \\
\hline PAR & $\begin{array}{l}\text { Parafuso autoa- } \\
\text { tarraxante }\end{array}$ & $\begin{array}{c}\text { Rothoblaas } \\
\text { HBS } 8180\end{array}$ & 6 & $\begin{array}{c}\text { Diâmetro: } 8 \mathrm{~mm} \\
\mathrm{~L}_{\max }: 180 \mathrm{~mm} \\
\mathrm{~L}_{\text {rosc. }}: 80 \mathrm{~mm} \\
\end{array}$ \\
\hline Encaixe C30 & $\begin{array}{l}\text { Encaixe de ma- } \\
\text { deira } \\
\end{array}$ & E. pellita & 6 & D: retangular $30 \mathrm{~mm}$ x $30 \mathrm{~mm}$ x $30 \mathrm{~mm}$ \\
\hline Encaixe $\mathrm{C} 45$ & $\begin{array}{l}\text { Encaixe de ma- } \\
\text { deira }\end{array}$ & E. pellita & 6 & D: retangular $30 \mathrm{~mm}$ x $30 \mathrm{~mm}$ x $45 \mathrm{~mm}$ \\
\hline
\end{tabular}

$\mathrm{N}$ : número de corpos de prova; $\mathrm{D}$ : dimensões; $\mathrm{L}_{\max }$ : Comprimento total; $\mathrm{L}_{\mathrm{rosc}}$ : Comprimento rosqueado.

\subsection{Método do teste}

Os ensaios foram realizados em máquina universal eletromecânica digital Instron EMIC com capacidade máxima de $200 \mathrm{kN}$. Foi utilizada uma cantoneira metálica no centro da peça horizontal do corpo de prova para medição de deformações com relógio comparador. Uma chapa metálica com $28 \mathrm{~mm}$ de espessura foi colocada no topo da barra horizontal para distribuir a carga aplicada na prensa.

O procedimento de carregamento empregado no ensaio seguiu a previsão existente no projeto de norma ABNT PN 02:126.10-001-4 [22], que é baseado na norma internacional ISO 6891 [23]. O procedimento consiste na aplicação de carga monotônica com dois ciclos de carga e descarga para a acomodação da ligação antes de aplicação de carga crescente até a ruptura. O procedimento recomenda que ligações altamente deformáveis devem ter o ensaio finalizado quando o deslocamento do corpo de prova chegar a $15 \mathrm{~mm}$. O arranjo dos corpos de prova na prensa universal está apresentado na Figura 3.

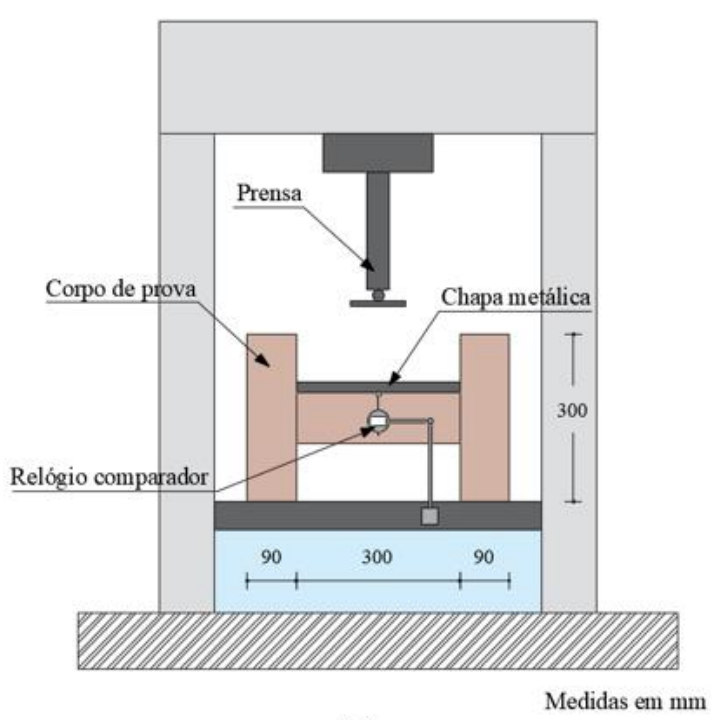

(a)

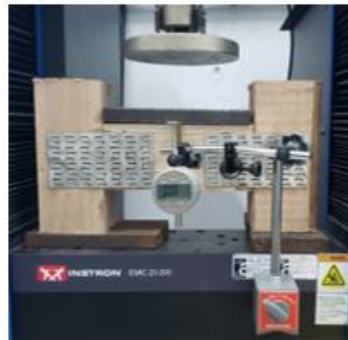

(b)

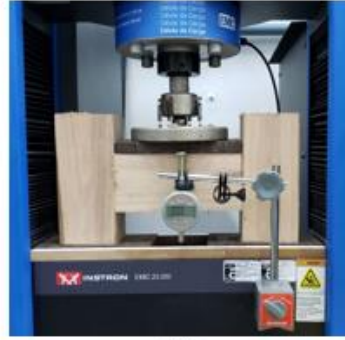

(c)

Figura 3: Arranjo de corpo de prova para ensaio resistência ao cisalhamento das ligações: (a) desenho esquemático do ensaio; (b) corpo de prova com chapa de dente estampado e (c) corpo de prova com parafuso autoatarraxante.

Para a comparação entre os resultados de resistência obtido nos ensaios de cada grupo de ligações com chapa de dente estampado e com encaixe de madeira foi utilizado o teste de análise de variância (ANOVA), acompanhando de teste Tukey para 95\% de significância. Para a normalidade dos dados encontrados foi utilizado o teste de Shapiro-Wilk. Os testes estatísticos foram feitos com o software PAST. 


\section{RESULTADOS}

A Tabela 2 apresenta os valores médios de força máxima cisalhante das ligações $\left(\mathrm{f}_{\mathrm{v}, \max }, \mathrm{em} \mathrm{kN}\right)$, o número de amostras $(\mathrm{N})$, a umidade da madeira no momento do ensaio (U, em \%), o desvio padrão (DP), o coeficiente de variação $(\mathrm{CV}$, em \%) e os valores mínimos e máximo para cada grupo. Foi encontrado um baixo coeficiente de variação para todos os grupos $(\mathrm{CV}<18 \%)$, indicando pouca dispersão e alta confiabilidade dos dados.

Tabela 2: Resistência ao cisalhamento de ligações de madeira de pequena seção.

\begin{tabular}{l|c|c|c|c|c|c|c}
\hline \multicolumn{1}{c|}{ LIGAÇÃo } & $\mathbf{N}$ & $\mathbf{U}(\%)$ & $\begin{array}{c}\mathbf{f}_{\mathbf{v}, \max } \\
\mathbf{( k N )}\end{array}$ & $\mathbf{D P}$ & $\begin{array}{c}\mathbf{C V} \\
(\%)\end{array}$ & $\begin{array}{c}\text { MíN. } \\
\mathbf{( k N )}\end{array}$ & $\begin{array}{c}\text { MÁX. } \\
(\mathbf{k N})\end{array}$ \\
\hline CDE-ACA & 5 & 16,40 & $38,10 \mathrm{~b}$ & 2,58 & 6,77 & 34,41 & 40,00 \\
\hline CDE CCC & 5 & 17,00 & $36,46 \mathrm{bc}$ & 1,18 & 3,26 & 34,84 & 37,63 \\
\hline CDE-AAA & 5 & 17,20 & $34,35 \mathrm{c}$ & 2,02 & 5,90 & 32,12 & 37,62 \\
\hline PAR & 6 & 16,50 & 18,00 & 0,00 & 0,00 & 18,00 & 18,00 \\
\hline Encaixe C30 & 6 & 16,80 & $18,92 \mathrm{a}$ & 1,71 & 9,08 & 16,11 & 20,00 \\
\hline Encaixe C45 & 6 & 17,10 & $47,73 \mathrm{~d}$ & 3,04 & 6,38 & 42,67 & 50,00 \\
\hline
\end{tabular}

\section{DISCUSSÃO}

A umidade da madeira estava em equilíbrio com o ambiente como esperado para regiões com umidade relativa do ar entre $75 \%$ e $85 \%$. De acordo com a formulação proposta pela ABNT NBR 7190 [9], a resistência pode ser até $15 \%$ maior que a encontrada para a condição de umidade da madeira próxima a 12\%, como esperado em outras regiões do Brasil que se encontram com umidade relativa do ar inferior a $65 \%$. A relação de umidade da madeira e resistência das ligações é discutida por HARADA et al. [24], onde os autores mostram que a resistência de ligações metálicas (parafuso e chapa de dente estampado) aumenta entre $1 \%$ e $3 \%$ para cada ponto percentual de umidade que a madeira seca em serviço. Optou-se por não utilizar fórmulas de correção da resistência para umidade padrão $(U=12 \%)$, pois a pesquisa objetivou apresentar resultados realistas para cidades que tenham umidade relativa ao ar parecida com a de Salvador, onde a pesquisa foi conduzida.

Para as ligações com encaixe, não foi encontrada referência que relacionasse a umidade com a resistência ao cisalhamento, no entanto, cabe mencionar que a variação de umidade influência a resistência de embutimento da conexão, não sendo, essa questão, abordada na presente investigação. Mesmo assim, vale ressaltar que para esta pesquisa não foi verificada a ocorrência de folga das ligações por encaixe devido a eventual variação de umidade.

Nos ensaios com chapa de dente estampado foi encontrada uma carga média máxima $\left(\mathrm{F}_{\mathrm{v}, \max }\right)$ de 36,30 $\mathrm{kN}$ considerando todos os grupos testados, dentro do intervalo de significância para $95 \%$ dos dados. A Figura 4 mostra a curva carga versus deslocamento para cada um dos grupos ensaiados com chapa de dente estampado. A falência da ligação aconteceu majoritariamente por escoamento e ruptura da chapa (Figura 4c), sendo registrada falência por arrancamento da chapa (Figura 4d) em dois corpos de prova. Não foi registrada ruptura na madeira em nenhum dos corpos de prova. 


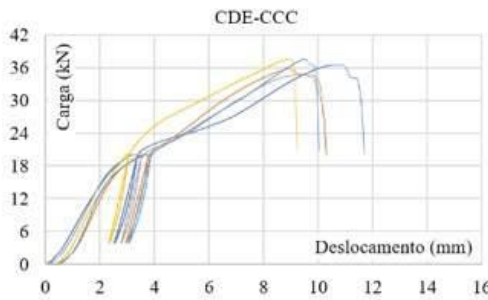

(a)

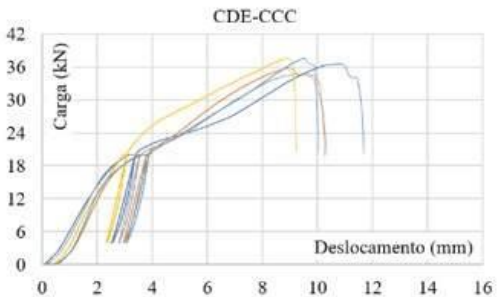

(b)

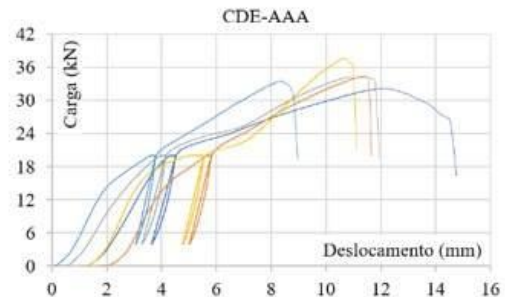

(c)

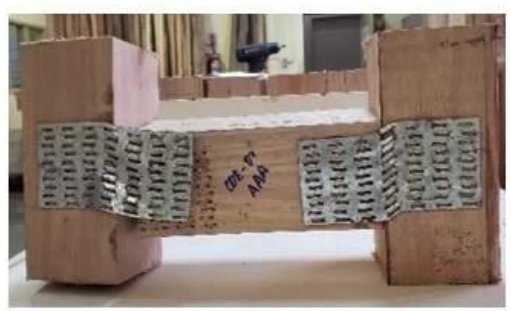

(d)

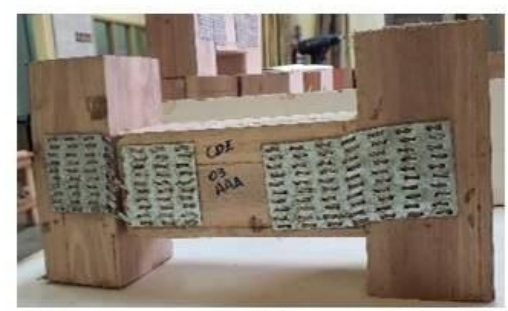

(e)

Figura 4: Curva carga versus deslocamento para cada grupo de ensaio com chapa de dente estampado e tipo de falência da ligação: (a) grupo CDE-ACA; (b) grupo CDE-CCC; (c) grupo CDE-AAA; (d) falência por arrancamento da chapa; (e) falência por escoamento e ruptura da chapa de dente estampado.

Durante o ensaio foi percebido que alguns corpos de prova, cujas faces conectadas apresentaram presença de cerne (CDE-ACA e CDE-CCC), tinham resistência ao cisalhamento 11\% superior quando comparadas às que eram ligados com faces de presença apenas de alburno (CDE-AAA). O ensaio permitiu inferir que a presença de madeira mais dura (cerne) na ancoragem dos dentes da chapa dentada confere maior resistência à ligação do que em regiões mais macias (alburno e madeira juvenil). Apesar da diferença estatística, todos os resultados obtidos para o conjunto dos corpos de prova encontraram-se dentro de uma mesma ordem de grandeza, próximos aos valores da média.

As características anatômicas e a diferença de umidade da madeira também podem explicar essa variação da resistência, quando conectadas em diferentes regiões da madeira. Logo, pode-se concluir que, apesar de os resultados indicarem pequena diferença da resistência de cisalhamento para ligações com presença de cerne, o experimento não pode ser conclusivo em afirmar se a densidade da madeira foi o fator que definiu essa variação, uma vez que uma quantidade maior ou menor de cerne e alburno modificam essa propriedade. Outro fator interveniente que deve ser analisado nesse processo é a anisotropia da madeira, como evidenciado por WAHRHAFTIG [25], uma vez que essa propriedade pode alterar os modos de ruptura do material.

Nos ensaios realizados com parafuso autoatarraxante não se observou ruptura do parafuso. A falha da ligação foi definida pelas deformações excessivas da peça horizontal, conforme estabelecido pelo procedimento normativo (deslocamento $>15 \mathrm{~mm}$ ). Devido à ruptura dúctil da ligação, foi considerada que a carga máxima do ensaio seria de $18 \mathrm{kN}$ para todos os corpos de prova. Ressalta-se que todos os corpos de prova atingiram a deformação máxima considerada para o fim do ensaio, o que correspondeu a uma força aplicada em torno de $15 \mathrm{kN}$, como demonstrado na curva de carga versus deslocamento presente na Figura 5. A análise do gráfico da Figura 5 permite observar que ocorreu a plastificação da madeira na região de contato com o parafuso, ainda na fase de deformação elástica desse, permitindo a rotação da peça horizontal e contribuindo para falência da ligação.

A falha das ligações por parafuso autoatarraxante foi determinada pela flexão do parafuso na peça e ruptura da madeira no entorno do furo, assim como previsto pelo modo 3 da Teoria de JOHANSEN [7]. Foi percebido que no valor correspondente a $10 \%$ da carga estimada $\left(\mathrm{f}_{10 \%}=1,8 \mathrm{kN}\right)$ houve um afrouxamento da ligação devido a pequenas fraturas da madeira. Esse aspecto pode ser observado na resposta obtida no ensaio quando se obtém um relativo aumento do deslocamento para uma pequena variação da força aplicada. Este resultado está evidenciado com uma seta em vermelho na curva da Figura 5. 

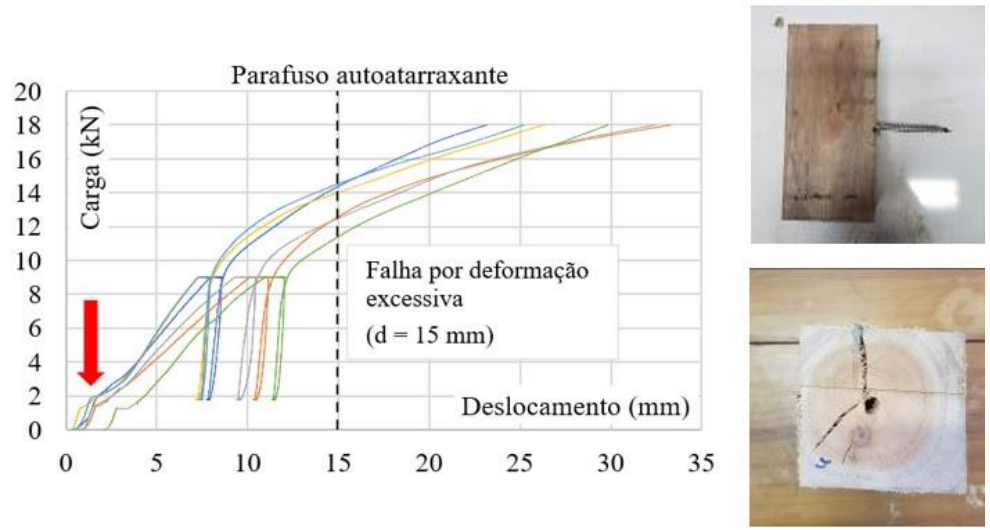

Figura 5: Curva carga versus deslocamento para ensaio com parafuso autoatarraxante e condição da ligação após ruptura.

A baixa resistência desse tipo de ligação deve-se ao fato de que a ancoragem do parafuso ter sido feita na região da medula, pois, sendo essa a parte mais frágil da madeira, propicia o aparecimento de rachaduras. O limite de proporcionalidade da ligação ocorreu no instante em que $50 \%$ da carga estimada $\left(\mathrm{f}_{50 \%}=9 \mathrm{kN}\right)$ foi alcançada, o que pode ser percebido pelo fato de que o ciclo de carga e recarga no ensaio não se sobrepõem.

Tendo em vista que essa ligação é pouco rígida e altamente deformável, é recomendado o uso de pelo menos dois parafusos para limitar a rotação da peça. No entanto, tomou-se como proposta desse trabalho o estudo de uma prática de obra, prevista no manual para obras em wood frame da Diretriz SINAT No 05 [20], onde os parafusos são utilizados com ângulo de $90^{\circ}$ em relação às fibras da madeira.

Na Figura 6 está presente a curva carga versus deslocamento para as ligações de encaixe de madeira para os grupos Encaixe C30 e Encaixe C45. A característica da curva de cada corpo de prova do grupo Encaixe C30 mostra que a falência da ligação por encaixe ocorre com a flexão do pino. Após a primeira ruptura, salientada com seta em vermelho na Figura 6, o comportamento da madeira é seguido pela compressão perpendicular às fibras, caracterizado pelo aumento de resistência e aumento considerável da deformação, este comportamento é esperado para encaixes do tipo retangular [26]. Em seguida, a ligação é acompanhada de múltiplas fraturas, sendo considerado como falência da ligação o momento da primeira ruptura, conforme indicado pela seta em vermelho.

Para o grupo Encaixe C45 foi identificado um comportamento similar ao grupo anterior, sendo mais evidente o momento em que ocorre a compressão perpendicular às fibras. Nesse caso, pôde ser percebido um aumento considerável da deformação relacionada a um pequeno aumento da resistência. Portanto, é crível entender que a falência da ligação foi estabelecida pela carga correspondente ao patamar da curva carga versus deslocamento. De um modo geral, pode-se afirmar que a falência da ligação ocorreu quando a deformação do conjunto, medida pelo relógio comparador, aproximava-se de $5 \mathrm{~mm}$ para o grupo C30 e de $8 \mathrm{~mm}$ para o grupo $\mathrm{C} 45$.

HU e GUAN [27] encontraram similar comportamento para a curva carga versus deslocamento para encaixes circulares. CLAUS, SEIM e SCHRÖDER [28] registram que a execução de chanfro nos encaixes retangulares redistribui as tensões na ligação, no entanto, mencionam que os resultados encontrados nos ensaios não apresentam melhorias significativas. A ligação, portanto, conforme resultados encontrados na presente investigação, estão em consonância com o trabalho dos mencionados pesquisadores, apresenta comportamento dúctil, que é o comportamento desejado para as estruturas de madeira. 


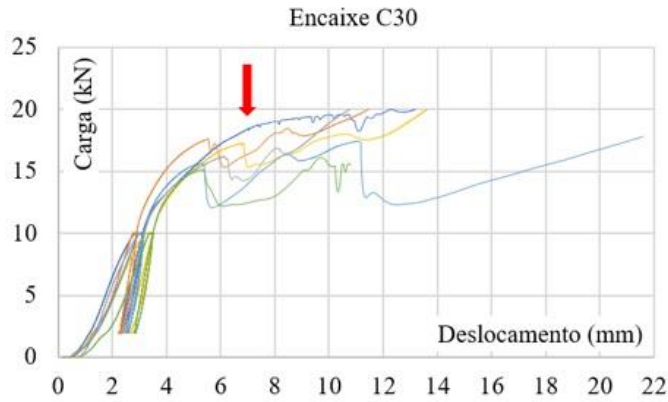

(a)

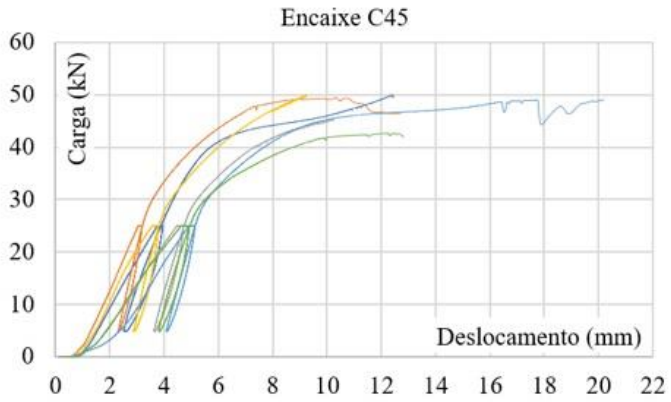

(b)

Figura 6: Curva carga versus deslocamento para ensaio com encaixe de madeira.

Com os ensaios realizados foi possível concluir que a rigidez de ligações do tipo encaixe retangular não é aumentada de acordo com variações dimensionais da ligação. No entanto, quando se observam os resultados dos ensaios do grupo C45 verifica-se haver, em média, uma resistência maior que o dobro das apresentadas pelo grupo C30, demostrando que o aumento do pino na direção longitudinal contribui de forma significativa para aumento de resistência. WU et al. [29] puderam comprovar que o aumento na direção longitudinal do pino, em ligações por encaixe retangulares, contribui exponencialmente para a resistência da ligação. AKCAY, ECKELMAN e HAVIAROVA [30] verificaram que, no caso de pinos circulares, o aumento na direção longitudinal do pino também conduz a um aumento significativo na resistência da ligação, quando comparado com o aumento da seção transversal do pino.

Pela análise dos resultados obtidos é possível verificar que a ligação com encaixe com dimensões de 30 mm x 30 mm x 45 mm (Encaixe C45) obteve os melhores resultados de resistência ao cisalhamento e rigidez. Em segundo lugar vem a ligação de chapa de dente estampado, seguida da ligação com encaixe com dimensão $30 \mathrm{~mm}$ x $30 \mathrm{~mm}$ x $30 \mathrm{~mm}$ (Encaixe C30) e aquela realizada com parafuso autoatarraxante. Adicionalmente aos aspectos de resistência e rigidez, as ligações por encaixe apresentam a vantagem de não necessitarem da completação de elementos metálicos, importante propriedade quando se deseja evitar a corrosão de ambientes agressivos como os encontrados no litoral brasileiro [31] e melhorar a durabilidade da ligação [32]

ECKELMAN, AKCAY e HAVIAROVA [33] construíram uma pequena residência de wood frame com montantes de seção $89 \mathrm{~mm}$ x $89 \mathrm{~mm}$, conectados com ligações do tipo encaixe circular. Em seu trabalho, os autores deixam evidente que a confecção de ligações por encaixe proporciona benefícios ao processo construtivo pela eficiência na industrialização devido à velocidade na execução das peças. Vale ressaltar, ainda, que a proposta de ligação por encaixe de forma retangular é a mais rápida de ser executada pois demanda equipamentos mais simples que a circular, além de ter maior resistência [34].

Para se obter uma maior eficiência na execução das ligações do tipo encaixe, recomenda-se o uso de máquina de corte com sistema de controle computacional ou Computer Numeric Control (CNC) o que permite maior velocidade de operação e maior precisão de corte. Caso se usem cavilhas é importante salientar a necessidade de estar a cavilha completamente seca, ou seja, isenta de qualquer umidade, a fim de se evitar problemas devido a retração da madeira, além de erros devido ao mal posicionamento do furo.

\section{CONCLUSÕES}

Neste trabalho, avaliou-se a resistência de ligações do tipo chapa de dente estampado, parafuso autoatarraxante e encaixe de madeira para madeira de eucalipto jovem destinada para a produção de painéis do sistema wood frame. Para isso, foi utilizada a madeira de Eucalyptus pellita classe C20 com idade variando entre 7 e 10 anos de idade. O estudo das ligações mostrou-se importante para a evolução nas pesquisas de adaptação da madeira de eucalipto jovem para produção de painéis do sistema wood frame. A partir da realização deste trabalho, foi possível concluir que:

1. a ligação com chapa de dentes estampados apresentou ganho na capacidade resistente média de $11 \%$, quando os dentes da chapa são ancorados em madeira de cerne;

2. em comparação com as outras ligações estudadas, as ligações realizadas com chapas de dente estampado apresentaram boa resistência mecânica e rigidez. No entanto, antes que seja definido o uso desse tipo de ligação devem ser avaliados os riscos de oxidação da chapa metálica em face do seu emprego em locais com algum nível de agressividade ambiental;

3. as ligações por parafuso autoatarraxante com cravação a $90^{\circ}$ foram as que apresentaram resultados menos favoráveis. Isso se dá em razão de terem baixa resistência ao cisalhamento e reduzida 
rigidez quando comparadas com as outras ligações propostas (chapa de dente estampado e encaixes). Portanto, esse tipo de ligação é recomendado apenas para a conexão de travessas;

4. na ligação aparafusada foi notado que a ruptura da união ocorreu por plastificação da madeira na região do furo, causando a rotação da peça horizontal e deformação excessiva do corpo de prova. Não se recomenda, devido a esse aspecto, a utilização de apenas um parafuso na ligação entre montantes e banzos;

5. as ligações do tipo encaixe apresentaram melhor resistência ao cisalhamento, assim como maior rigidez. No entanto, ressalta-se a importância do uso de técnicas computacionais de corte como forma de melhor a qualidade da confecção da ligação.

\section{AGRADECIMENTOS}

Os autores agradecem à Coordenação de Aperfeiçoamento de Pessoal de Nível Superior (CAPES) pela bolsa de pesquisa, ao Laboratório de Ensaios em Durabilidade dos Materiais (LEDMa) e Laboratório de Madeiras (LabMad) da Universidade Federal da Bahia pela cessão de suas infraestruturas.

\section{BIBLIOGRAFIA}

[1] ARAUJO V., VASCONCELOS, J., CORTEZ-BARBOSA, J., et al., "Wood consumption and fixations of carbon dioxide and carbon from timber housing techniques: A Brazilian panorama". Energy \& Buildings, v. 216, Jun. 2020.

[2] BASZEŃ, M., "Semi-rigid Behavior of Joints in Wood Light-frame Structures". Procedia Engineering, v. 172, p. 88-95, 2017.

[3] FOREST PRODUCTS LABORATORY., Wood Handbook: Wood as an Engineering Material, Madison, US Department of Agriculture (USDA), Forest Service, 2010.

[4] ROSA, C. C., "Influência de temperaturas elevadas e do ângulo de inserção do parafuso na resistência ao arrancamento de parafusos autoatarraxantes", Tese de D.Sc., Centro Tecnologico/UFSC, Florianopolis, SC, Brasil, 2018.

[5] VALLE, A., MORAES, P. D., MANTOVANI, G., et al., "Influence of temperature on the adhesion of fibre reinforced polymers to timber surface". Ambiente Construído, v. 19, n. 3, p. 25-38, Jun. 2019.

[6] CALIL NETO, C., MOLINA, J., CALIL JUNIOR, C., et al., "Modelagem numérica do comportamento de ligações com parafusos auto-atarraxantes em X em corpos de prova de MLC com madeiras do tipo Eucalipto urograndis". Revista Matéria, v. 33, n. 01, Mai. 2017.

[7] JOHANSEN, K. W., "Theory of Timber Connections", IABSE: International Association of Bridge and Structural Engineering, v. 9, p. 249-262, 1949.

[8] LIU, Y., XIONG, H., "Lateral performance of a semi-rigid timber frame structure : theoretical analysis and experimental study", Journal of Wood Science, n. 64, p. 591-600, Jun. 2018.

[9] ASSOCIAÇÃO BRASILEIRA DE NORMAS TÉCNICAS - ABNT, "NBR 7190: Projeto de estruturas de madeira", Rio de Janeiro, 1997.

[10] SERVIÇO FLORESTAL BRASILEIRO - SNIF, Boletim do Sistema Nacional de Informações Florestais, 1 ed., Brasília, Ministério do Meio Ambiente, 2017.

[11] PAIVA FILHO, J. C., ALMEIDA, L. A., CASTRO, V. et al., "Diagnóstico do uso da madeira como material de construção no município de Mossoró-RN/Brasil”, Revista Matéria, v. 23, n. 3, Out. 2018.

[12] ALMEIDA, D., DIAS, A. "Resistência da madeira ao embutimento perpendicular às fibras: comparação de métodos de ensaio", Ambiente Construído, v. 19, n. 4, p. 175-181, Out./Dez. 2019.

[13] CARRASCO, E., SMITS, M., MANTILLA, J. "Resistência ao cisalhamento da ligação bambu-bambu: Influência da pressão de colagem”. Revista Matéria, v. 22, 2017.

[14] ALSMARKER, T., BRÜNINGHOFF, S., WINTER, S., "Diaphragms and bracing", In: Blass, H. J.; Sandhass, S. (eds), Timber Engineering - Principles for Design, ed. 1, chapter D.9, Karlsruhe, Alemanha, KIT Scientific Publishing, 2017.

[15] SINHA, A., "Postpeak residual capacity of nailed connections of a shear wall", Holzforschung, v. 68, n. 8, p. 987-992, Dez. 2014.

[16] WANG, R., WEI, S.; LI, Z. et al., "Performance of connection system used in lightweight glubam shear wall”, Construction and Building Materials, v. 206, p. 419-431, 2019. 
[17] ALINOORI, F.; MOSHIRI, F.; SHARAFI, P. et al., "Reinforcement methods for compression perpendicular to grain in top/bottom plates of light timber frames", Construction and Building Materials, v. 231, p. 116377, Jan. 2020.

[18] SILVA, J. S., "Parâmetros construtivos para painéis verticais adaptados do sistema wood frame em madeira de eucalipto jovem”, Dissertação de M.Sc. Escola Politécnica/UFBA, Salvador, BA, Brasil, 2018.

[19] INSTITUTO BRASILEIRO DE ÁRVORES (IBÁ), “Relatório Anual IBÁ 2019”, Brasilia, 2019.

[20] SISTEMA NACIONAL DE AVALIAÇÕES TÉCNICAS, "Diretriz SINAT No005 Revisão 2: Sistemas construtivos estruturados em peças leves de madeira maciça serrada, com fechamentos em chapas (Sistemas leves tipo "light wood frame")", Brasilia, 2017.

[21] SANTOS, F. L., SENA, C. S., SILVA, G. D. et al., "Avaliação da estabilidade dimensional de madeira de Eucalyptus pellita". In: VI CBCTEM - Congresso Brasileiro de Ciências e Tecnologia da Madeira, Santarém, Pará, Brasil, Out. 2019.

[22] ASSOCIAÇÃO BRASILEIRA DE NORMAS TÉCNICAS - ABNT. "PN 02:126.10-001-4: Método de ensaio para determinação da resistência e da rigidez de ligações com conectores mecânicos em estruturas de madeira", Rio de Janeiro, 2017.

[23] INTERNATIONAL STANDARD ORGANAZATION - ISO, "ISO 6819: Timber structures — Joints made with mechanical fasteners - General principles for the determination of strength and deformation characteristics", Genebra, 1981.

[24] HARADA, M., HAYASHI, Y., HAYASHI, T. et al. "Effect of moisture content of members on mechanical properties of timber joints". Journal of Wood Science, v. 51, n. 3, p. 282-285, 2005.

[25] WAHRHAFTIG, A. M., "Experimental exploratory study of failure modes in models of wooden rectangular beams of Hymenae sp", Revista Matéria, v. 23, p. 1-14, Mar. 2018.

[26] WU, G., GONG, M., GONG, Y. et al. "Mechanical performance of mortise and tenon joints prereinforced with slot-in bamboo scrimber plates". Journal of Wood Sciences, v. 65, n. 38, 2019.

[27] HU, W.; GUAN, H., "A finite element model of semi-rigid mortise-and-tenon joint considering glue line and friction coefficient", Journal of Wood Science, v. 65, n. 1, 2019.

[28] CLAUS, T.; SEIM, W.; SCHRÖDER, B., "Multiple tenons - Experimental study on load-bearing capacity and deformation characteristics", In: World Conference on Timber Engineering. Vienna, Austria, Ago. 2016.

[29] WU, G., ZHONG, Y., GONG, Y. et al., "Application of modern wood product glulam in timber frame with tenon-mortise joints and its structural behavior", Journal of Renewable Materials, v. 7, n. 5, p. 451-461, 2019.

[30] AKCAY, H., ECKELMAN, C., HAVIARONA, E. "Withdrawal, shear, and bending moment capacities of round mortise and tenon timber framing joints", Forest Products Journal, v. 55, n. 6, p. 60-67, 2005.

[31] WAHARFTIG, A. M., CARVALHO, R. F., "Design and Construction of Wooden Structure to Replace Collapsed Steel Structure", Practice Periodical on Structural Design and Construction (ASCE), v. 21, n. 3, 2016.

[32] POLETTI, E., VASCONCELOS, G., BRANCO, J. M. et al. "Effects of extreme environmental exposure conditions on the mechanical behaviour of traditional carpentry joints", Construction and Building Materials, v. 213, p. 61-78, 2019.

[33] ECKELMAN, C. A.; AKCAY, H.; HAVIAROVA, E., "Performance tests of small barn frame constructed with round mortise and tenon joints", Forest Products Journal, v. 56, n. 4, p. 41-47, 2006.

[34] TANKUT, A.; TANKUT, N., "The effects of joint forms (Shape) and dimensions on the strengths of mortise and tenon joints", Turkish Journal of Agriculture and Forestry, v. 29, n. 6, p. 493-498, 2005.

\section{ORCID}

Filipe Luigi Dantas Lima Santos

Júlia Cruz da Silva

Rita Dione Araújo Cunha

Alexandre de Macêdo Wahrhaftig

Sandro Fábio César https://orcid.org/0000-0002-4557-2630

https://orcid.org/0000-0002-2591-0065

https://orcid.org/0000-0001-9372-6879

https://orcid.org/0000-0002-7144-1917

https://orcid.org/0000-0002-8606-3576 